\title{
The Digital Protection and Practice of Intangible Cultural Heritage Crafts in the context of New Technology
}

\author{
Jue $\mathrm{Wu}^{1,2}$ a , Licai Guo ${ }^{2}$, Jialin Jiang ${ }^{2}$, Youfu Sun ${ }^{1}$ * \\ ${ }^{1}$ College of wood science and technology, Nanjing Forestry University, Nangjing, Jiangshu, China \\ ${ }^{2}$ The Academy of Art and Design Zhejiang Sci-Tech University, Hangzhou, Zhejiang, China
}

\begin{abstract}
Based on the protection and inheritance of intangible cultural heritage crafts, this paper takes Dongyang bamboo weaving as an example, introduces Virtual Reality (VR), 3D digital programming and other new digital technologies to the protection and inheritance of bamboo weaving crafts. From the three following aspects: experience, application and infection, this paper summarizes the digital protection and inheritance methods and processes of intangible cultural heritage, finds out the meeting point with the modern consumer market, and makes the cultural heritage "live" in the present and into the future.It helps to better understand the traditional crafts, promote the inheritance and development of bamboo weaving crafts and provide references for the protection and inheritance of intangible cultural heritage.
\end{abstract}

\section{Background}

Bamboo weaving art is an ancient and magical handicraft art, known as "the miracle in the history of weaving". After the development of more than 6000 years, there are more than 1000 kinds of bamboo weaving crafts and patterns now. However, with the acceleration of the pace of contemporary life, the rapid change of production mode and the impact of modern industrial products, the production of bamboo weaving products is shrinking and may die out; Moreover, there is no successor in the inheritance of bamboo weaving crafts, and many bamboo weaving crafts are facing loss with the death of old artists. As a kind of traditional handicraft, bamboo weaving craft, including a large number of tacit knowledge, exists in the minds of inheritors. Many amazing crafts in traditional weaving will be a pity if they disappear in the long history with the death of old artists. Therefore, it is of great significance and value to think about how to excavate the value of traditional crafts and preserve crafts in the context of new digital technology.

Many scholars at home and abroad have done a lot of research in the field of the inheritance and protection of intangible cultural heritage. The existing digital protection mainly focuses on document sorting and database information terminal construction. Aiming at bamboo weaving, the traditional intangible cultural technique, this paper puts forward the digital protection and inheritance methods and strategies to make users understand the traditional intangible bamboo weaving crafts better, promote the inheritance and development of bamboo weaving crafts and provide reference for the protection and inheritance of intangible cultural heritage.

\section{Digital Technology}

In the current digital environment of the integration of culture and technology, a new round of information technology revolution represented by Internet, big data, artificial intelligence, cloud computing and other technologies is surging. The development of technology is an important driving force for the digitization of intangible cultural heritage. The new content carrier, production mode, cultural experience and interactive scene generated by digital technology promote the transformation and dissemination of intangible cultural resources ${ }^{[]}$. As for the technologies, it mainly uses database technology, digital image technology, three-dimensional technology (3D), virtual reality (VR), augmented reality (AR), digital animation technology, motion capture technology and so on.

\subsection{Digital library technology}

Digital library technology is to sort out, summarize, classify, analyze and summarize the collected survey data, and then sort, sequence and input the materials, so that the data can be comprehensively, completely and truly recorded. In the post-processing, each step will be recorded in sections, and the tools used in each step will be recorded one by one, and then the process flow can be clearly visualized according to the name and number of the steps. Then, using the computer network platform to upload the results of field investigation together with static and dynamic digital data to form a database, realize the

\footnotetext{
aj5277@zstu.edu.cn

b270754565@qq.com

c1004406398@qq.com

dyfsun@njfu.edu.cn
} 
preservation and sharing of resources, improve the efficiency of research work and save research costs.

\subsection{New Media Technology}

New media technology is a relative concept, which is the double result of the development of science technologies and social culture ${ }^{[2]}$.By making full use of advanced technologies of image, audio, video, geographic information system, 3D animation, etc. together with the characteristics and advantages of digital media and the construction of interactive three-dimensional communication channels, it can digitize the relevant cultural heritage accurately and carefully. It can not only transform the transmission of intangible cultural heritage from plane communication to interactive 3D communication, but promote the content of intangible cultural heritage communication from superficiality, pattern and marginalization to depth as well, presenting the culture of intangible cultural heritage in an all-round way, deeping the communication connotation of intangible cultural heritage, and making the precious cultural heritage of mankind more widely learned, researched and inherited. For example, network-based communication can expand the influence and wide spread of intangible cultural heritage, transform the intangible cultural heritage from regional culture to a wider range of culture, strengthen cultural communication, and has extremely vital significance to the active promotion of the protection and dissemination of national intangible cultural heritage.

\subsection{Virtual Reality Technology}

Virtual reality is a kind of new world created by computer and electronic technology, which is a seemingly real simulation environment. Through a variety of sensing devices, users can follow their own feelings to get involved, using human natural skills to observe and operate the virtual world. At the same time, virtual reality provides intuitive and natural real-time perception of vision, hearing and touching, and makes the participants "immerse" in the simulation environment ${ }^{[3]}$. In recent years, virtual interactive technology has been widely used in the field of intangible cultural heritage protection, which is mainly divided into two areas: interactive experiences between users and the environment and interactive experiences of users' behaviors.

\subsection{Computer Aided Technology}

Computer aided technology, also called CAD, is carrying out various creative activities in the field of design with the support of computer and its corresponding CAD system $^{[4]}$.Compared with traditional design, CAD has undergone qualitative changes in design methods, design process, design quality and efficiency. Its fundamental purpose is to effectively support the innovative design of products $^{[5]}$. Nowadays, the main applications of CAD in the field of intangible cultural heritage are digital software customization and artificial intelligence generation.

\section{Digital Protection Path of the Crafts of Intangible Cultural Heritage}

Thinking from the three-level value of cultural connotation of intangible cultural heritage crafts, this paper puts forward the combination with new digital technology, which is suitable for the inheritance and protection path of bamboo weaving crafts from three aspects of experience, application and infection (Figure 1).

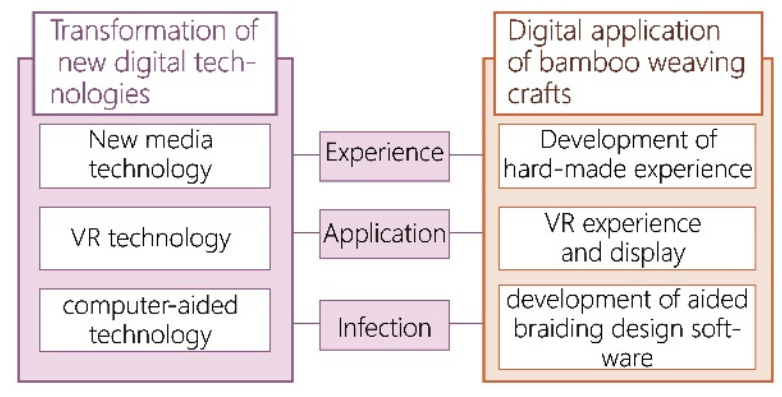

Figure 1 Digital protection and inheritance path of crafts

\subsection{New experience of intangible cultural}

In the era of experience economy, experience value is emphasized and users are willing to enjoy paying for the whole experience process provided by products ${ }^{[6]}$. Nowadays experience is the new source of value in today's society. The study of weaving crafts originally emphasizes running-in of a lot of practice, which is now providing rich contents and materials for experience economy. By experiencing the production process, users can better feel the wisdom of traditional creation, deepen their feelings with traditional intangible cultural heritage, and improve their recognition of handcrafts.

\subsection{Modern production application of intangible cultural heritage crafts}

Let traditional crafts nourish modern design. There are mathematical laws in bamboo patterns. Through digital technology and parametric software, bamboo patterns can be transformed into programs. Users and designers can flexibly match and obtain personalized weaving patterns products without long-term training. Through the construction of parametric weaving pattern database, bamboo weaving can be a new design language, which can be better used in various household products.

\subsection{Resonance of craftsman spirit in intangible cultural heritage}

The design concept of intangible cultural heritage crafts is included in the production process. Every seemingly repeated process actually requires the craftsman to make judgments and choices on the current situation. The more highly dependent on the individual experience of the handicraft category, the more it requires a higher degree of integration of production and design ${ }^{[7]}$. The basic forms of craft inheritance are: the inheritance of father and son, oral transmission, master apprentice teaching and teaching by 
precept and example. In the training process, rich and dynamic situations are needed, and the designers and producers are required to have high situational sensitivity, familiarity and flexibility.

The integration of VR, AR and reality is changing the form of interaction, which helps to create a perceptual and situational environment, and helps to understand and learn tacit knowledge. With the daily use of technology, the physiological, emotional and cognitive behaviors of each technology user is profoundly reshaped. Lucie Smith optimistically predicts that as human society enters the machine age, handicrafts will be gradually changing from practical art to spiritual art. The new interactive system, as an aesthetic work to communicate the soul, will realize the integration of design and technology again ${ }^{[8]}$.

\section{Digital Practice of Bamboo Weaving}

Through field visits to bamboo weaving masters and literature research, the research group deeply studies the traditional patterns and works of bamboo weaving, and tries a variety of digital technologies to study the effective forms of expression suitable for bamboo weaving crafts.

\subsection{Experience Project of Bamboo Weaving Crafts}

In the process of traditional bamboo weaving experience, the bamboo strips are often soft and not easy to control. There will be problems in the picking and pressing process such as skewing, warping and deformation, leading to failure. Beginners are easy to break up patterns in the process of picking and pressing at the beginning of the experience. The experimenters said that the production process was too time-consuming and labor-consuming, there were few things that could be made by themselves, and the products were not practical. This kind of bamboo weaving experience was not a cultural edification for the experiencer, but more of a boring process.

From the perspective of experience, the research group studies the multiple contact points of users' expectation and experience, and finally designs the "Bian Ying" experience package, as shown in Figure 2. The material experience package is composed of a set of weaving AIDS and material packages. Users can define and design their own products, even novices can learn and weave regular hexagon texture in a short time. Through the auxiliary tools of hexagonal weaving, we can not only weave the derived textures of double hexagonal weaving and snowflake weaving, but also make it convenient for young users to make DIY patterns and weave their own textures.

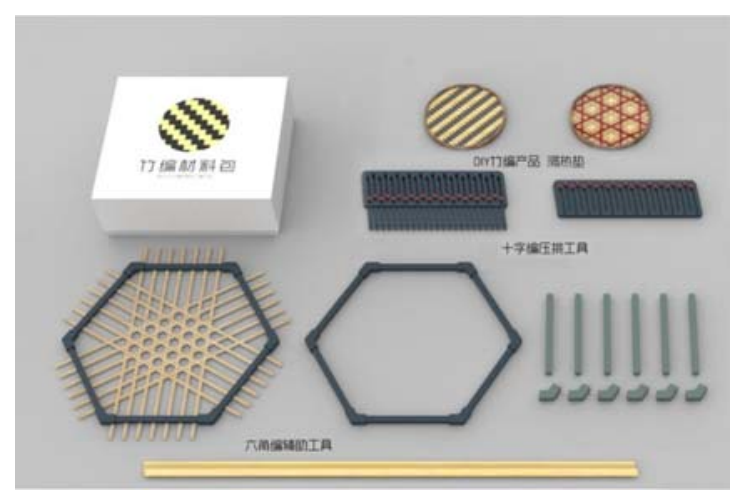

Figure 2 "Editing and Mapping" experience package

\subsection{Virtual Game Based on Somatosensory Interaction}

Through interactive display system, the digital interactive technology and gamification elements are added into the digital design of intangible cultural heritage bamboo weaving, so as to build an interactive learning environment for the public, improve the interaction between learners and intangible cultural heritage bamboo weaving. It strives to show visitors the charm of bamboo weaving culture, stimulate their browsing interest, and provide visitors with a unique interaction experience and arouse teenagers' interests in learning.

The team uses the Kinect somatosensory interaction engine or processing of Unity 3D to establish an interactive device for bamboo weaving virtual experience, as shown in Figure 3. Through the cooperation of simple words or sound guidance, users can learn simple weaving cases through gesture interaction. The audience can participate in the process of creating crafts manually, and experience the technology and crafts of bamboo weaving in the virtual environment, so that more people can appreciate the artistic charm and cultural value of traditional bamboo weaving crafts.

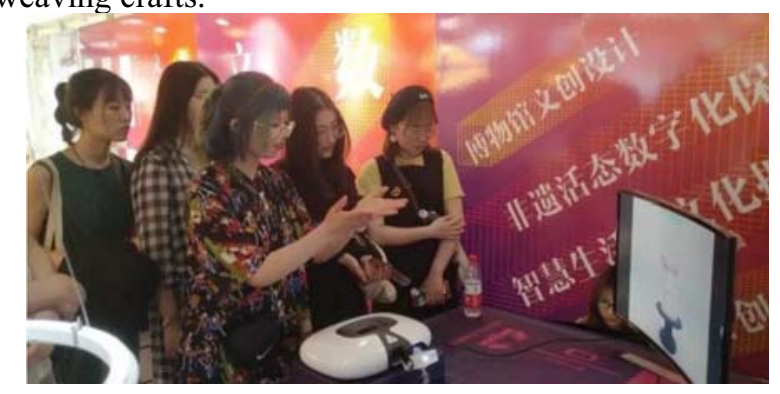

Figure 3 Digital bamboo weaving virtual

\subsection{Parametric Customization System for Bamboo Weaving Products}

Based on the previous research on bamboo weaving, according to different weaving effects, the research group extracts the warp and weft line data as variables, and weaves patterns with Grasshopper. Among them, the warp and weft density, braided thread spacing, line width, color and other parameters can be freely adjusted by users. The finished program can also be flexibly applied to different types of products or shapes to quickly generate braided 
effect skin. Parametric design greatly improves the modeling efficiency, and users can control the relevant parameters more conveniently. In addition, consumers can not only buy designers' elaborate works on the website, but also obtain personalized products through the app on the online website, as shown in Figure 4. On the online website, users can freely choose the type, shape, size, material, color and production method of the products to be customized on the online website. The website will show the final effect picture of the product in real time. After the user confirms the final printing effect of the product, the user can select the packaging and distribution method, either picking it up from the store or delivering it by express delivery is ok. The final product will be delivered to the user quickly. Through various ways of Internet promotions, more young people will participate in the understanding of bamboo culture and design their own personalized bamboo products.

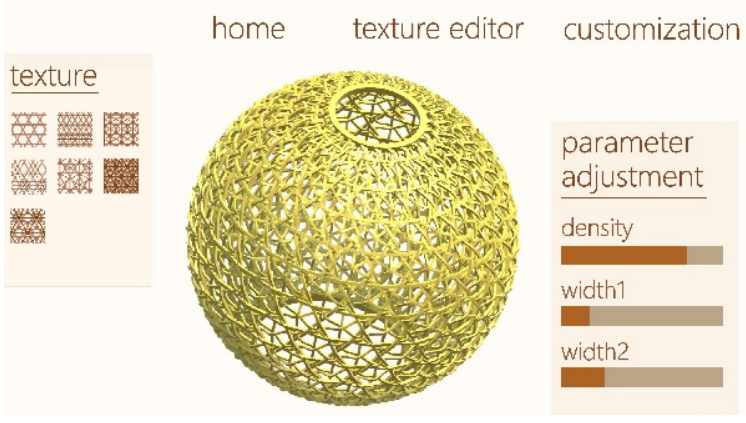

Figure 4 Customization platform of parametric products

\section{Conclusion and Expectation}

Intangible cultural heritage is the record of the traditional memory and cultural genes of various Chinese nationalities. It not only embodies the wisdom and spirits of a nation, but also reflects the unique cultural form and aesthetic ideal of a nation. It is an important source of national cohesion. Taking bamboo weaving as an example, this paper summarizes and puts forward the digital protection and inheritance methods and the process of intangible cultural heritage, moreover, introduces new digital technologies such as Internet, 3D, AR and VR into the protection and inheritance of bamboo weaving crafts, which provides a certain reference for the protection and inheritance of intangible cultural heritage. Looking forward to more innovation and integration of digital technology in the future, traditional intangible cultural heritage crafts will be into modern life, providing new ideas for the protection and inheritance of intangible cultural heritage.

\section{REFERENCES}

1. Jiang Youyu, Min Xiaolei (2018). Endogenous creative system based on rural cultural resources. Decoration, (4), 34-38.

2. SunYijun, Wang Jianlei (2012). On the influence and promotion of new media on cultural communication. Contemporary communication, (1), 46-48.
3. Jiang Xuezhi, Li Zhonghua (2004). Present situation of VR technology at home and abroad. Journal of Liaoning Technical University, (2), 238-240.

4. Pan Yunhe, Sun Shouqian, Bao Enwei (1999). The developing situation and trend of computer aided industrial design technology. Journal of ComputerAided Design and Computer Graphics,(3),3-5.

5. Sun Shouqian, Bao Enwei, Chen Zheng, Pan Yunhe (1999). Research status and development trend of computer-aided conceptual design. China Mechanical Engineering, (6), 3-5.

6. Belk RW (1975).Situational Variables and Consumer Behavior. Journal of Consumer Research, (2)

7. Wu Weiyi (2020). Research on the creative psychology and social aesthetic connotation of new technology and artificial intelligence design. Industrial engineering design, 2(1), 26-32.

8. LUCIE S E,1981 The Story of Craft: the Craftsman's Role in Society. Cornell University Press, New York, the United States. 